\title{
The value of intraoperative neurophysiological monitoring for microsurgical removal of conus medullaris lipomas: a 12-year retrospective cohort study
}

Olaf Suess $^{1 *}$, Sven Mularski ${ }^{1}$, Marcus A Czabanka², Mario Cabraja ${ }^{3}$, Stefanie Hammersen ${ }^{4}$ and Theodoros Kombos ${ }^{5}$

\begin{abstract}
Background: Lipomas in the lower spinal canal can lead to progressive neurological deficits, so they may have to be surgically removed. Intraoperative neurophysiological monitoring serves to minimize the morbidity of the surgical procedure. However, so far there are no evidence-based recommendations which type of monitoring procedure or combination of procedures to choose.

Methods: The aim of this study was to evaluate the feasibility and value of various intraoperative monitoring techniques: motor and sensory evoked potentials (MEP, SEP), free-running and triggered electromyography (EMG). Thirty cases of spinal lipomas of the Conus medullaris (dorsal Type A: 20.0\%; caudal Type B: 33.3\%; transitional Type C: 46.7\%) were retrospectively evaluated over a 12-year period.

Results: The patients were mostly pediatric and suffered from persistent pain (73.3\%), pareses (56.7\%), sensory deficits (43.4\%), and/or urogenital dysfunctions (60.0\%). SEPs were successfully evoked in $66.7 \%$ of cases, MEPs in $86.7 \%$ of cases, and EMGs in 100\%. MEP alterations correlated with direct mechanical maneuvers in the operating site. SEP changes correlated mostly with physiological events, such as rinsing/cooling of the operating site. Spike-, burst- or tonic train-activity was found in the free-running EMG that occurred only with certain manipulation patterns. Irreversible MEP changes and signal loss in the triggered EMG correlated with post-operative deficits.

Conclusions: The results of this study showed, that intraoperative monitoring could be considered a helpful tool during lipoma tumor surgery near the Conus medullaris. Most reliable results were obtained from transcranial MEPs, free-running EMGs, and triggered EMGs. That's why the authors favor a routine set-up consisting of at least these three techniques, as this enables mapping at the beginning of the operation, continuous functional testing during surgery, and prognosis of the post-operative symptomology.
\end{abstract}

Keywords: Intraoperative monitoring, Neurophysiological monitoring, Spinal lipoma, Conus medullaris, Microneurosurgery

\section{Background}

Spinal lipomas are benign tumors of the spinal canal. They consist of up to $90 \%$ fat cells but can also contain fibrous, muscular, neural, and other embryogenic tissue. Lipomas are differentiated clinically, pathohistologically, and ontogenically into several types: purely epidural lipomas, purely intradural lipomas, lipomas of the Conus

\footnotetext{
*Correspondence: o.suess@drk-kliniken-berlin.de

'Zentrum für Wirbelsäulenchirurgie und Neurotraumatologie, DRK Kliniken Berlin Westend, Spandauer Damm 130, 14050 Berlin, Germany

Full list of author information is available at the end of the article
}

medullaris, lipomas of the Filum terminale, and spinal angiolipomas [1]. Lipomas are one of the main reasons for tethering of the spinal cord (Figure 1), which can lead to progredient neurological deficits, such as pareses, sensory deficits, and bladder or colon dysfunction [1-4].

Lipomas of the Conus medullaris are the most common form. They occur with an incidence of 1 in 4000 births in Western countries, and they have a 2:3 male:female ratio $[1,5]$. They are categorized into three subgroups, depending upon where they adhere to the Conus: a) dorsal, b) caudal, and c) transitional (Figure 2) $[1,2,4,5]$. Lipomas of the 


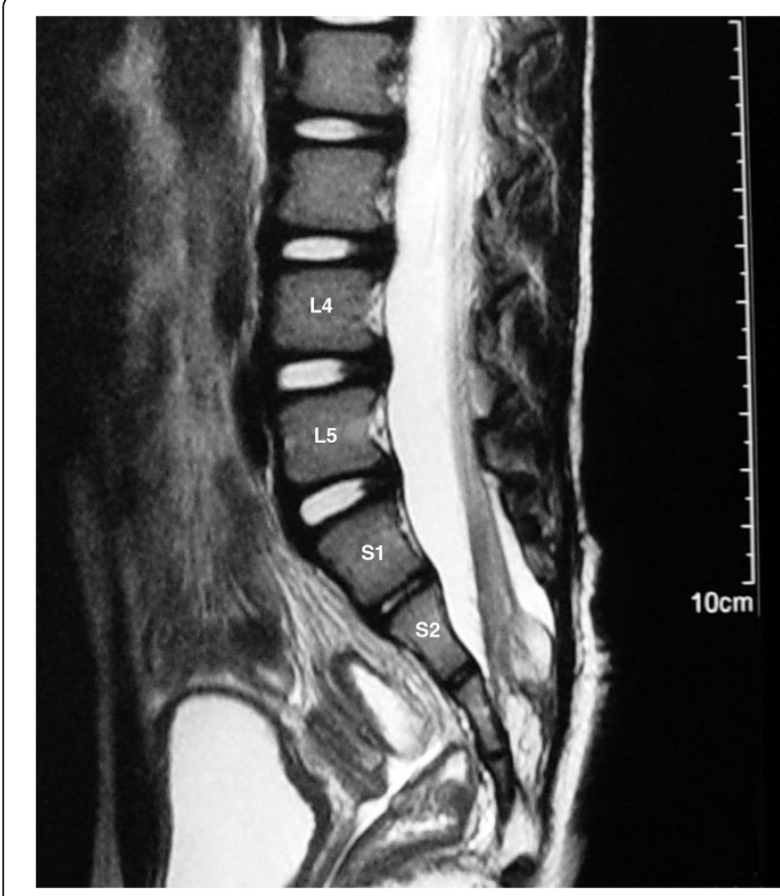

Figure 1 Sagital MRI, T2-weighted, of a lumbosacral lipoma with Conus base at level S2 and consecutive tethering at level S3, in a 12 year old female patient.

Conus medullaris are diagnosed as early as a mean age of 6 months, because a soft, subcutaneous mass of fat is palpable in the lumbar vertebral area already by this age in $90 \%$ of the children [1]. Further symptoms caused by the lipoma of the Conus medullaris include skin changes (such as hypertrichosis), capillary hemangiomas, and neuroorthopedic deformities of the extremities and feet due to asymmetrical innervation $[1,3,5,6]$. The neurological symptoms are mostly sensory and motor deficits of the lower extremities, limited or lost bladder and colon control, and back pain (arising mostly later with age) [1-3].

Neurosurgical intervention removes the tumor and untethers the nerve roots, in order to improve neurological deficits and prevent further progression of the pathology. There is an surgical morbidity of up to $24 \%$, because the tumor adheres strongly to the spinal cord and the exiting nerve roots and/or because the tumor tissue invades into the dura $[7,8]$. Various neurophysiological examination techniques are used to reduce the risk of iatrogenic neurological deficits in spinal-cord surgery [9]. These techniques serve to detect nerve tissue in the operating site and to monitor its function until the end of the operation. Motor evoked potentials (MEPs) have been used to monitor descending pathways. After transcranial electric or magnetic stimulation, muscle response potentials can be recorded electromyographically from defined muscles of the extremities [3,10-13]. Somatosensory evoked potentials (SEPs) are suitable for monitoring ascending pathways. Surface stimulation is delivered over the N. tibialis, and potentials are recorded cranially via scalp electrodes [10-14]. Free-running and triggered electromyography (EMG) are two methods for directly testing nerve fibers in the operation site [15-17]. The continuous monitoring of the free-running EMG will show any mechanical manipulations of the nerve roots (e.g. during tumor removal). The direct electrical stimulation of triggered EMG can be used to test the transmission capacity of nerve roots.

So far there are no evidence-based recommendations on the choice of the best and most reliable monitoring procedure or combination of procedures. The aim of this study was to evaluate the various monitoring procedures for their effectiveness and prognostic value when used during neurosurgical treatment of spinal lipomas. This report provides an evaluation of each of the procedures and estimation on the best combination.

\section{Methods}

\section{Study design}

This is a retrospective analysis of data gathered at two clinics (1. Department of Neurosurgery, Charité University Hospital, Berlin, Germany and 2. The Spine \& Neurotrauma

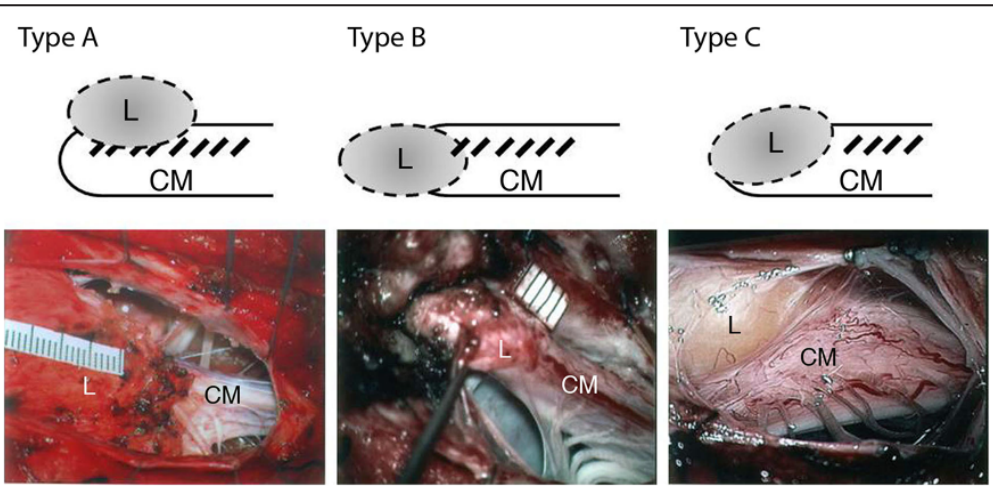

Figure 2 Distribution of the lipomas (L) of the Conus medullaris (CM) according to their attachment spot: type A - dorsal, type B - caudal, type C - transitional. 
Center, DRK Kliniken Berlin Westend, Berlin, Germany) between January 1, 2000 and December 31, 2012.

\section{Patients}

Patients were included in the analysis who had to undergo a neurosurgical operation for a lipoma in the area of the Conus medullaris under intraoperative MEP/SEP/EMG monitoring. Data evaluation was performed on the basis of patient records and digital recordings of the monitoring results from the Department's Neurophysiological Examination Unit. Cases were excluded from the analysis if they had incomplete recordings of the preoperative or postoperative neurological status or gaps in the monitoring recordings.

During the investigation period, a total of 35 cases of neurosurgical operations for Conus medullaris lipomas were identified, and 30 of these 35 cases remained evaluable after application of the exclusion criteria. Of these 30 cases, 19 (63.3\%) were female. The patients had a median (range) age of 17.6 years $(0.5-51$ years).

\section{Tumor localization}

The lipoma was attached dorsally to the Conus medullaris in 6 of the 30 cases (20.0\%) (Figure 2A). The lipoma was caudal in 10 cases (33.3\%) (Figure 2B). The lipoma had grown transitionally in 14 cases $(46.7 \%)$ (Figure $2 \mathrm{C}$ ).

\section{Clinical symptoms}

Preoperatively, 22 of the 30 patients $(73.3 \%)$ suffered from persistent local pain (VAS $>3 / 10$ for at least 3 months) at the time of the diagnosis. Pain grading in children was assessed using the "Wong-Baker Faces scale" for children aged 3 years and older, and using "CRIES-grading" for children under the age of three. Another 18 patients (60.0\%) reported urogenital dysfunctions (bladder and/or colon). Seventeen patients (56.7\%) presented pareses of BMRC (British Medical Research Council grading, 1978) grade $4 / 5$ or worse. Thirteen patients (43.3\%) reported dermatome-specific sensory deficits. In another 11 cases (36.7\%), neuro-orthopedic deformities were found in the physical exam. These symptoms were found either as single key symptoms or in combinations of various more mildly pronounced complaints (Table 1). Four of the patients $(13.3 \%)$ were clinically asymptomatic. These were all 6-10 month old infants who were noticed because of a palpable soft-tissue lumbar swelling and skin stigmata.

\section{Operation, anesthesia, and monitoring}

The operations were carried out with the goal of removal the tumor as completely as possible and untethering the dural sac. The operating technique was microsurgical in all cases. The surgical procedures were carried out by three different surgeons.

\begin{tabular}{ll}
$\begin{array}{l}\text { Table } 1 \text { Sociodemographic and clinical characteristics of } \\
\text { the patients }\end{array}$ \\
\hline Number of cases & 30 \\
Sex (f/m) & $19 / 11$ (ratio = 1.7:1) \\
Age & median: 17.6 years \\
Tumor localization & \\
Type A (dorsal) & $6(20.0 \%)$ \\
Type B (caudal) & $10(33.3 \%)$ \\
Type C (transitional) & $14(46.7 \%)$ \\
Clinical symptomalogy at diagnosis*: & \\
Pain (VAS >3/10 for at least 3 months) & $22(73.3 \%)$ \\
Urogenital dysfunction (bladder/colon) & $18(60.0 \%)$ \\
Pareses (BMRC $\leq 4 / 5)$ & $17(56.7 \%)$ \\
Sensory deficits & $13(43.3 \%)$ \\
Neuroorthopedic deformities & $11(36.7 \%)$ \\
Clinically asymptomatic (only stigmata) & $4(13.3 \%)$ \\
\hline
\end{tabular}

${ }^{*}=$ as single symptom or in combination with other symptoms.

All surgeries were carried out under total intravenous anesthesia with Propofol/Remifentanil, and muscle relaxants were only used for narcosis induction, in order to assure undisturbed monitoring. The degree of intraoperative muscle relaxation was documented through continuous "Train of Four - TOF” measurement.

The intraoperative neurophysiological monitoring was conducted with a Viking or Endeavor system (Nicolet Biomedical/Viasys Healthcare; Madison, WI, USA). Data were evaluated online by four different persons during the timeperiod of the study and stored digitally for further offline analysis.

\section{Motor evoked potentials}

Motor potentials were evoked by means of a transcranial high-frequency cortex stimulation in multipulse technique (Table 2). Scalp screw electrodes were placed into the head skin, above the motor cortex at $\mathrm{Cz}$ (anode) and $6 \mathrm{~cm}$ frontal from $\mathrm{Cz}$ (cathode). The stimulation frequency was $500 \mathrm{~Hz}$ with a train of 5, an impulse duration of $0.3 \mathrm{~ms}$, and stimulation intensity of no more than $100 \mathrm{~mA}$. The evoked compound muscle action potentials (CMAPs) from different muscle groups were recorded with 12 subdermal needle electrodes arranged pairwise (20-37 mm/27-28 gauge). Recording electrodes were placed above the $M$. vastus lateralis, the $M$. tibialis anterior, the M. peronaeus longus, the M. gastrocnemius, and the sphincter ani in quadrants respectively (Table 2). For evaluation, the signal was amplified 10000x and displayed with a filter setting of 100-1500 Hz. The stimulation intensity was increased stepwise until at least all 6 muscle groups unilaterally showed MEP activity or the upper limit of $100 \mathrm{~mA}$ was reached. 
Table 2 Stimulation and recording parameters for the MEP, SEP, and EMG monitoring

\begin{tabular}{|c|c|c|c|c|}
\hline & MEP Monitoring & SEP Monitoring & $\begin{array}{l}\text { Electromyography } \\
\text { (free-running) }\end{array}$ & $\begin{array}{l}\text { Electromyography } \\
\text { (triggered) }\end{array}$ \\
\hline Stimulation point & $\begin{array}{l}\mathrm{Cz} \text { (anode) } / \mathrm{Cz}+6 \mathrm{~cm} \\
\text { (cathode) }\end{array}$ & $\begin{array}{l}\text { Posterior N. tibialis (bilaterally) } \\
\text { cathode: proximally between } \\
\text { Achilles tendon und Malleolus } \\
\text { medialis anode: } 2-3 \mathrm{~cm} \text { distal }\end{array}$ & $-1-$ & direct nerve root stimulation \\
\hline $\begin{array}{l}\text { Stimulation } \\
\text { technique }\end{array}$ & $\begin{array}{l}\text { transcranial, high frequency } \\
\text { ("multipulse-technique) } \\
\text { scalp-screw electrodes }\end{array}$ & adhesion electrodes & $-/-$ & $\begin{array}{l}\text { bipolar stimulation probe, } \\
\text { monophasic square impulse }\end{array}$ \\
\hline $\begin{array}{l}\text { Stimulation } \\
\text { frequency }\end{array}$ & $500 \mathrm{~Hz}$, "train of 5" & $4.7 \mathrm{~Hz}$ & $-/-$ & $3.0 \mathrm{~Hz}$ \\
\hline Impulse duration & $0.3 \mathrm{~ms}$ & $0.2 \mathrm{~ms}$ & $-/-$ & $0.1 \mathrm{~ms}$ \\
\hline Stimulation intensity & $\max .100 \mathrm{~mA}$ & $\max .60 \mathrm{~mA}$ & $-/-$ & $\max .2 \mathrm{~mA}$ \\
\hline Recording technique & $\begin{array}{l}\text { bipolar, subdermal needle } \\
\text { electrodes }\end{array}$ & scalp-screw electrodes & $\begin{array}{l}\text { bipolar, subdermal needle } \\
\text { electrodes }\end{array}$ & $\begin{array}{l}\text { bipolar, subdermal needle } \\
\text { electrodes }\end{array}$ \\
\hline \multirow[t]{8}{*}{ Recording location } & M. vastus lateralis, & \multirow[t]{8}{*}{$C z^{\prime}-F p z$} & \multirow[t]{2}{*}{$(\mathrm{L} 2) / \mathrm{L} 3 /(\mathrm{L} 4)-\mathrm{M}$. vastus lateralis, } & \multirow{2}{*}{$\begin{array}{l}(\mathrm{L} 2) / \mathrm{L} 3 /(\mathrm{L} 4)-\mathrm{M} \text {. vastus } \\
\text { lateralis, }\end{array}$} \\
\hline & M. tibialis anterior, & & & \\
\hline & M. peronaeus longus, & & \multirow[t]{2}{*}{$L 4 /(L 5) /(S 1)-M$. tibialis anterior, } & \multirow{2}{*}{$\begin{array}{l}\mathrm{L} 4 /(\mathrm{L} 5) /(\mathrm{S} 1)-\mathrm{M} . \text { tibialis } \\
\text { anterior, }\end{array}$} \\
\hline & M. gastrocnemius, & & & \\
\hline & M. sphincter ani QI/II, & & L5/(S1) - M. peronaeus longus, & L5/(S1) - M. peronaeus longus \\
\hline & M. sphincter ani QIII/IV & & S1 - M. gastrocnemius, & S1 - M. gastrocnemius, \\
\hline & & & $\mathrm{S} 2 / 3$ - M. sphincter ani QI/II, & S2/3 - M. sphincter ani QI/II, \\
\hline & & & S4/5 - M. sphincter ani QIII/IV & S4/5 - M. sphincter ani QIII/IV \\
\hline \multirow[t]{2}{*}{ Recording potential } & \multirow[t]{2}{*}{ CMAP } & P40/N50 & \multirow[t]{2}{*}{ multichannel EMG activity } & \multirow[t]{2}{*}{ multichannel EMG activity } \\
\hline & & (500-2000 signals averaged) & & \\
\hline Filter settings & $100-1500 \mathrm{~Hz}$ & $30-1000 \mathrm{~Hz}$ & $100-5000 \mathrm{~Hz}$ & $100-5000 \mathrm{~Hz}$ \\
\hline \multirow[t]{2}{*}{ Presentation } & visual & visual & auditory \& visual & auditory \& visual \\
\hline & $100 \mathrm{msec}, 200 \mu \mathrm{V} / \mathrm{div}$ & $100 \mathrm{msec}, 10 \mu \mathrm{V} / \mathrm{div}$ & 200-500 msec, 10-200 $\mu \mathrm{V} / \mathrm{div}$ & 200-500 msec, 10-200 $\mu \mathrm{V} / \mathrm{div}$ \\
\hline
\end{tabular}

\section{Somatosensory evoked potentials}

Somatosensory potentials were evoked by means of stimulation of the tibialis nerve. Surface adhering electrodes were placed above the distal course of the Nervus tibialis on the inner ankle. The stimulation frequency was $4.7 \mathrm{~Hz}$ in all cases with an impulse duration of $0.2 \mathrm{~ms}$ and a stimulus intensity of no more than $60 \mathrm{~mA}$. The recording took place by means of scalp electrodes above the sensory cortex at Cz'-Fpz. For evaluation, the N40/P50 potentials from at least 500 signals were averaged (500-2000) and displayed with a filter setting of $30-1000 \mathrm{~Hz}$.

\section{Electromyography}

A free-running EMG was recorded by means of subdermal needle electrodes, as described above for the MEPs. The filter setting was $100-5000 \mathrm{~Hz}$. The potentials were presented both visually on the monitor and audially over a loudspeaker system.

A bipolar stimulation-probe (Viasys Healthcare; Madison, WI, USA) was used for the triggered EMG. A monophasic square impulse with a stimulation frequency of $3.0 \mathrm{~Hz}$ and an impulse duration of $0.1 \mathrm{~ms}$ was used for nerve root stimulation. The individual nerve roots were stimulated directly in the area of their exit from the dural sac or after emergence from the tumor tissue, starting from $0 \mathrm{~mA}$, in $0.2 \mathrm{~mA}$ steps, until either a triggered potential was registered in the free-running EMG or the maximum stimulation intensity of $2 \mathrm{~mA}$ was reached. The recording electrode was placed above the $M$. vastus lateralis for the nerve roots (L2)/L3/(L4), above the $\mathrm{M}$. tibialis anterior for $\mathrm{L} 4 /(\mathrm{L} 5) /(\mathrm{S} 1)$, above the M. peronaeus longus for $\mathrm{L} 5 /(\mathrm{S} 1)$, above the M. gastrocnemius for S1, and above the sphincter ani in quadrants QI/II or QIII/IV for S2/3 and S4/5 respectively (Table 2). If an EMG potential was not evoked at the maximum stimulation, then the technical set-up and the relaxation status of the patient were checked, in order to rule out conduction disruptions as the explanation for the lack of response. The direct nerve-root stimulation took place: a) first for the localization of the nerve roots L3-S5 after opening of the dura and exposure of the lipoma, b) immediately before tumor removal, and c) at the end of the extirpation of the neoplasm for functional testing and prognostic evaluation. During the phase of tumor preparation, nerveroot-specific 
monitoring took place in intervals of approximately $30 \mathrm{sec}$, in order to differentiate between nerve tissue and tumor.

\section{Evaluation}

The evaluation of the monitoring results addressed the following three questions. 1) Which of the monitoring procedures allowed for reliable recording of potentials? 2) Were intraoperative maneuvers correlated with changes in the potentials registered? 3) Were intraoperative changes of potentials correlated with changes of the postoperative symptoms. Giving due consideration to natural physiological fluctuations, "obvious" MEP and SEP plots were defined as intraoperative amplitude reductions of more than 50\% (for MEP and SEP) or latency prolongations of more than $15 \%$ (for MEP).

\section{Results}

\section{Motor evoked potentials}

Monitoring of the MEP potentials was successful for at least one of the muscle groups mentioned in 26 of 30 cases (86.7\%) (Figure 3). In the other four cases, a CMAP response could not be generated in any of the discharged muscle groups, and this was viewed as a failure of the MEP technique. This correlated with a preexisting highgrade paraparesis (BMRC grade 2/5 distal from L3 bilaterally) in two cases. The other two cases were infants, 6 and 10 months old, without motor deficits.

In the 26 successful cases, potentials could be recorded in a mean of 8 of 12 monitored muscle groups. CMAPs could be generated most frequently and best from the M. gastrocnemius and the anterior M. tibialis, and thereafter (in descending order) from the M. peronaeus longus, the $M$. vastus lateralis, and the quadrants of the M. sphincter ani. The success of recording correlated negatively with preexisting pareses only for a BMRC of $2 / 5$ or worse $(\mathrm{r}=0.91 ; \mathrm{p}<0.005)$.

In 19 of these 26 cases (73.1\%), the MEP sequences remained uneventful during the entire operation. In the other 7 cases, $50-100 \%$ reductions of the amplitude of individual muscle groups were observed as direct correlates of an operative manipulation in the area of the Conus medullaris (traction, irrigation with unwarmed rinsing solution, perinerval coagulation). In 5 cases (19.2\%), these were reversible amplitude reductions of $50-80 \%$ during bipolar tumor preparation that resolved within a mean of 35 seconds after the electrocoagulation was stopped. Motor deterioration was observed immediately after the operation in one of these five cases, yet it was completely recovered by the 3-month post-op follow-up examination. An irreversible and lasting amplitude reduction of more than $80 \%$ was observed in 2 cases (7.7\%), in one muscle group each, after more aggressive preparation. In both cases, motor deterioration of at least 2 BMRC grades was observed for the affected muscle group (case 1: L5 musculature right-side from strength grade $4 / 5$ to $2 / 5$; case 2 : S1 musculature right-side from strength grade $4+/ 5$ to $2 /$ $5)$. These two cases of motor deterioration had not improved by the first follow-up visit 3-months post-op - in contrast to 3 other immediate postoperative reductions of motor strength in cases of uneventful or reversible potential courses, which all improved within a week post-op (Table 3).

\section{Sensory evoked potentials}

Monitoring of the tibial SEPs was successfully carried out in 20 of the 30 cases $(66.7 \%)$ (Table 3$)$. In the other 10 of 30 cases (33.3\%), an analyzable SEP could not be recorded even at the maximal stimulation intensity of
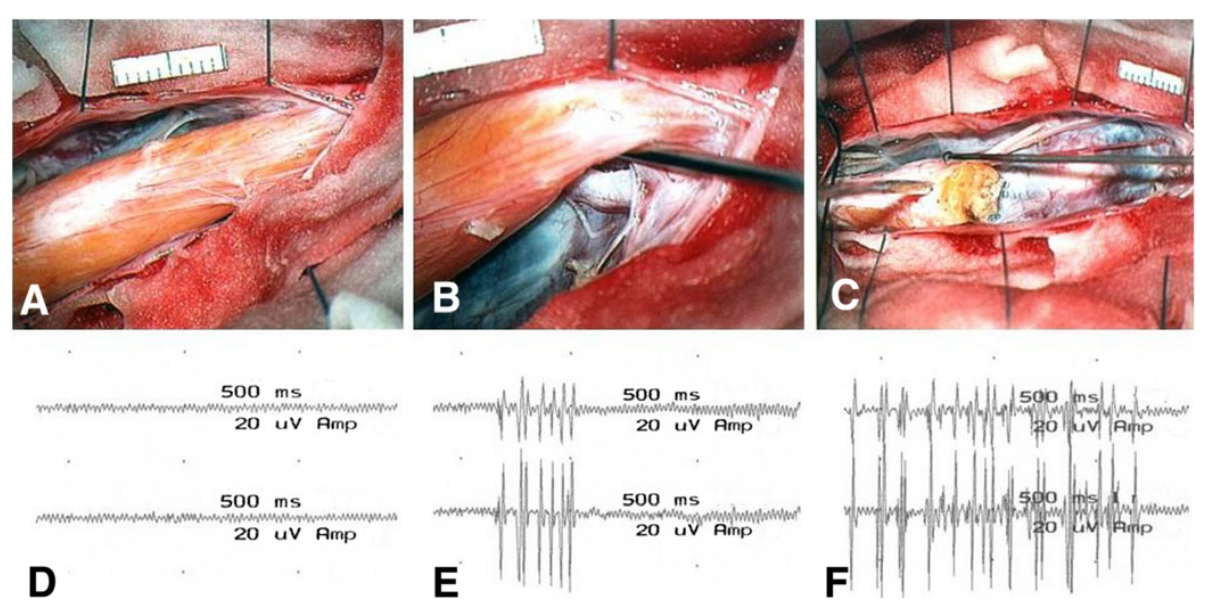

Figure 3 Results and noticeable events in the free-running EMG. EMG normal finding (D) for opened dura (A); "volley/burst" (E) from light contact (B); "train" activity (F) from stronger traction or after sharp tumor resection (C) close to neural structures. 
Table 3 Results of the MEP, SEP and EMG monitoring

\begin{tabular}{|c|c|c|c|c|c|c|c|c|}
\hline \multicolumn{9}{|c|}{ MEP $(n=30)$, technical feasibility: $86.7 \%(26 / 30)$} \\
\hline \multicolumn{5}{|c|}{ Motor strength - 24 hours post surgery } & \multicolumn{4}{|c|}{ motor strength -3 months post surgery } \\
\hline Intraoperative findings & + & $=$ & - & & + & $=$ & - & $\Sigma$ \\
\hline Uneventful & 1 & 16 & 2 & & 6 & 13 & 0 & $19(73.1 \%)$ \\
\hline Reversible & 0 & 4 & 1 & $\rightarrow$ & 1 & 4 & 0 & $5(19.2 \%)$ \\
\hline Irreversible & 0 & 0 & 2 & & 0 & 0 & 2 & $2(7.7 \%)$ \\
\hline \multicolumn{9}{|c|}{ SEP $(n=30)$, technical feasibility: $66.7 \%(20 / 30)$} \\
\hline \multicolumn{5}{|c|}{ Sensory status - 24 hours post surgery } & \multicolumn{4}{|c|}{ sensory status -3 months post surgery } \\
\hline Intraoperative findings & + & $=$ & - & & + & $=$ & - & $\Sigma$ \\
\hline Uneventful & 0 & 6 & 3 & & 1 & 5 & 3 & $9(45.0 \%)$ \\
\hline Reversible & 0 & 4 & 2 & $\rightarrow$ & 0 & 5 & 1 & $6(30.0 \%)$ \\
\hline Irreversible & 0 & 4 & 1 & & 0 & 5 & 0 & $5(25.0 \%)$ \\
\hline \multicolumn{9}{|c|}{ Free-run EMG $(n=30)$, technical feasibility: 100\% (30/30) } \\
\hline \multicolumn{5}{|c|}{ Motor strength -24 hours post surgery } & \multicolumn{4}{|c|}{ motor strength -3 months post surgery } \\
\hline Intraoperative findings & + & $=$ & - & & + & $=$ & - & $\Sigma$ \\
\hline Uneventful & 0 & 0 & 0 & & 0 & 0 & 0 & $0 / 30$ \\
\hline Bursts/salves & 2 & 22 & 6 & $\rightarrow$ & 8 & 20 & 2 & $30 / 30$ \\
\hline Trains & 0 & 4 & 6 & & 0 & 7 & 3 & $10 / 30$ \\
\hline \multicolumn{9}{|l|}{ Triggered EMG $(n=432)$} \\
\hline \multicolumn{4}{|l|}{ Before tx removal } & & & & \multicolumn{2}{|c|}{$\begin{array}{l}\text { after tx removal, before dura } \\
\text { closure }\end{array}$} \\
\hline Stimulation success & \multicolumn{3}{|c|}{ No. of nerve roots } & \multicolumn{3}{|c|}{ changes during tx removal } & \multicolumn{2}{|c|}{ No. of nerve roots } \\
\hline Yes & \multicolumn{3}{|c|}{$260(60.2 \%)$} & +81 & & & \multicolumn{2}{|c|}{$330(76.4 \%)$} \\
\hline No & \multicolumn{2}{|c|}{$172(39.8 \%)$} & $\rightarrow$ & -81 & & $\rightarrow$ & \multicolumn{2}{|c|}{$102(23.6 \%)$} \\
\hline
\end{tabular}

MEP: Correlation between the transcranial evoked MEP results (unchanged, reversible changes, irreversible changes) and the postoperative muscle strength, as a change in at least one of the recorded muscle groups L3-S5 (BMRC grade improved, unchanged, worse) within $24 \mathrm{~h}$ post-op and in the follow-up exam 3 months post-op. uneventful = amplitude and latency within individual limits; reversible potential changes (p.c.) = at least one and then no longer reaching these criteria at the latest by the end of the operation; irreversible p.c. = continuous exceeding of amplitude reduction $>50 \%$ or latency prolongation $>15 \%$ after spontaneous initiation.

SEP: Correlation between the tibialis SEP events (unchanged, reversible changes, irreversible changes) and the postoperative sensory status, as a subjective change in at least one dermatome L3-S5 (improved, unchanged, worse) within $24 \mathrm{~h}$ post-op and in the follow-up exam 3 months post-op. uneventful = amplitude and latency within individual limits; reversible p.c. = at least one and then no longer reaching these criteria at the latest by the end of the operation; irreversible p.c. $=$ continuous exceeding of amplitude reduction $>50 \%$ or latency prolongation $>15 \%$ after spontaneous initiation.

Free-run EMG: Correlation between the events of the free-running EMG (uneventful, volleys/bursts, trains) and the postoperative muscle strength, as a change in at least one of the recorded muscle groups L3-S5 (BMRC grade improved, unchanged, worse) within $24 \mathrm{~h}$ post-op and in the follow-up exam 3 month post-op. Triggered EMG: Number $(\mathrm{n})$ of the stimulated nerve roots and stimulation success before and after removal of the tumor (tx). ${ }^{*}=$ number of stimulation gains during the operation, ${ }^{* *}=$ number of stimulation losses during the operation.

$60 \mathrm{~mA}$. These 10 cases correlated neither with the age of the patients nor with the severity of preexisting sensory deficits.

In 9 of the successful 20 cases (45\%), the tibial SEP sequence remained uneventful during the entire operation, i.e. meaningful changes were not observed for either the amplitude or the latency. In the other 11 cases (55.0\%), reductions of the amplitude greater than $50 \%$ were observed, but they had no direct relation to the operative manipulations of the exposed nerve roots. A durable prolongation of the latency was not observed in any case. There was a lasting loss of potential in 5 cases - all after rinsing the operating site with unwarmed physiological saline. Only one of these 5 cases had a postoperative deterioration of the sensory status. In contrast, there were 3 cases with postoperative worsening of dermatome-specific deficits that during the operation had not shown any noticeable changes of the tibial SEP.

\section{Free-running electromyography}

As expected, a free-running EMG was recordable in all 30 cases (100\%) (Table 3, Figure 3). A "baseline noise" plotted with or without spontaneous activity was deemed to be a normal finding (Figure 3A,D). EMG signals in the form of repetitive polyphasic potential discharges (CRDs) without any corresponding surgical maneuver were to be found in $20 \%(6 / 30)$ of the cases immediately before dura opening. CRDs presented as waxing and waning highfrequency synchronous discharges with an amplitude 
range from $50 \mu \mathrm{V}$ to $1 \mathrm{mV}$. The discharge rate ranged from 10 to $100 / \mathrm{sec}$ with a discharge duration between 0.2 and 2.5 seconds. They resolved immediately after dura opening and were considered as non-pathologic. During the course of the operation, pathological EMG activity in the form of bi- or triphasic potentials with a large peak of up to $2500 \mu \mathrm{V}$ ("spike") or a whole complex of superimposed spikes ("burst") (Figure 3E) could be set off by direct contact of the nerve roots (Figure 3B). Spikes abruptly arose from and declined to baseline whereas bursts were longer in duration and lasted for up to 510 milliseconds. The overall spike/burst quotient was 1.8/1. The average number of spikes and bursts recorded per case was 73 . There was a $r=0.94$ correlation of these recordable phasic potentials to a mechanical manipulation as the trigger.

Lasting artifacts in the sense of tonic asynchronous potentials ("trains", Figure 3F) were set off by dragging or traction (Figure 3C). These potentials were characterized by a sudden onset with a fixed amplitude varying between 50 and $300 \mu \mathrm{V}$. The mean frequency was $152 \mathrm{~Hz}$ with a range of 50 to $330 \mathrm{~Hz}$. The trains lasted between $150 \mathrm{msec}$ and $4000 \mathrm{msec}$. The correlation of these tonic asynchronous potentials to mechanical maneuvers was $r=0.88$.

The occurrence of spikes or bursts was not significantly associated with the postoperative neurological symptoms, as spikes or bursts were documented at some timepoint in the operation or tumor preparation in all 30 cases. In contrast, an immediate post-operative worsening of motor deficits of more than one BMRC grade was to be found in 6 of the 10 cases with tonic "train" recordings. In 3 of these cases, the deterioration in motor strength was still unchanged at the 3-month follow-up. The other 3 regained the pre-operative motor status within the first 3 months after surgery, but without further improvement in the BMRC grading (Table 3). Thus, free-run EMG "train" activity detected all 5 cases with motor strength worsening that were detectable by the MEPs as well - plus in 1 of the 4 cases, where MEP monitoring was not feasible.

\section{Triggered electromyography}

The identification of spinal nerve roots was accomplished without technical problems in all 30 patients (100\%). Thus 16 nerve roots (L3-S5, bilaterally) per patient, in 30 patients, were stimulated, for a total of 432 nerve roots. In 260 of the $432(60.2 \%)$ stimulation attempts, it was possible to specifically identify nerve tissue and unambiguous map the musculature (Table 3; Figure 4A,B) after opening the spinal canal and before beginning the tumor removal. During the operative intervention, triggered EMG was used to identify nerve roots, e.g. within a tumor conglomerate. This enabled nerve tissue to be quickly, simply, and safely prepared free from tumor tissue growing around it. After removal of the tumor, 330 of the 432 (76.4\%) stimulation attempts were successful.

There were 81 gains of identification of nerve roots and11 losses of identification of nerve roots (Table 3 ). The gains were due to preparing free the nerve roots that were hidden in tumor conglomerates at the beginning of the operation. The losses of identification were accompanied by a postoperative deterioration of the BMRC
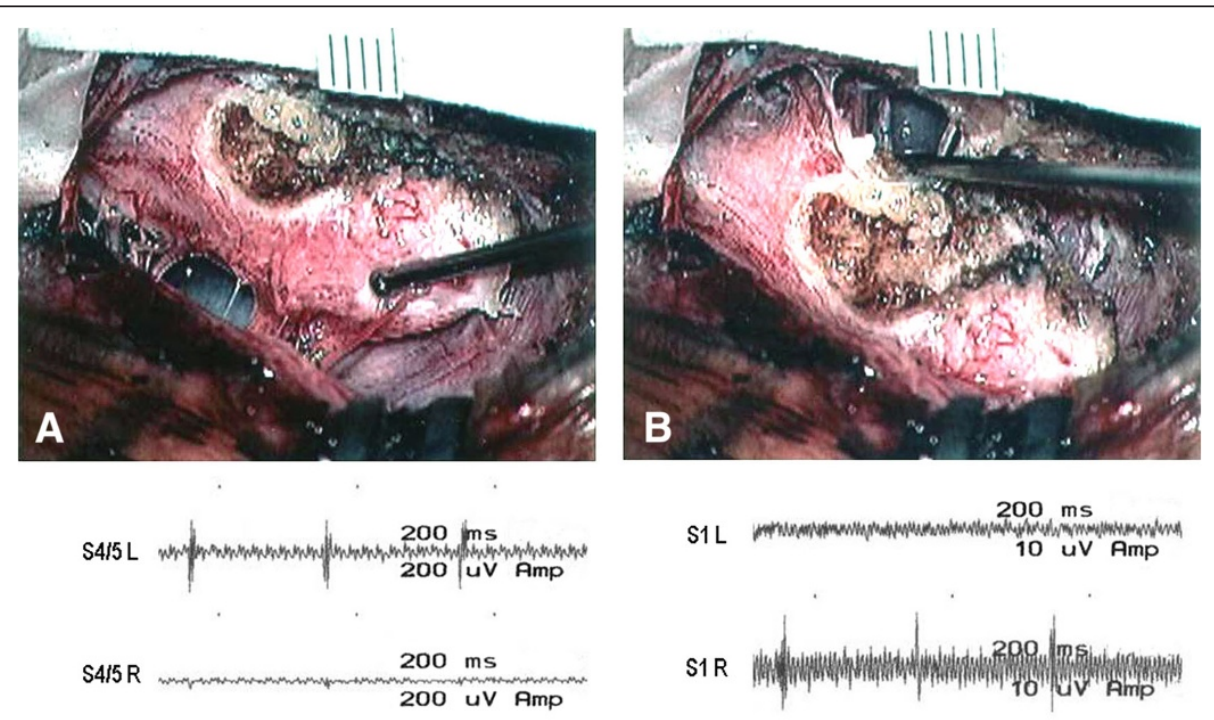

Figure 4 Results from the triggered EMG. Identification of the nerve roots S4/S5 left (A) and S1 right (B) during the incremental extirpation of the tumor. 
grade for the corresponding muscle group of at least 1 point in 3 cases. In another 2 cases, there was an intermittent bladder/colon dysfunction postoperatively. Gains of potential correlated with immediate post-operative clinical improvement of the motor function with only $r=0.41$.

\section{Discussion}

Spinal lipomas are the cause of tethering of the dura and the exiting nerve roots. Already in early childhood they can lead to several severe functional compromises such as pareses, sensory deficits, or urogenital dysfunctions $[1,3,6]$. Lipomas of the Conus medullaris are the most common form of spinal lipoma, and they have been often discussed in the literature [1,3-5]. There is broad consensus about the choice of treatment for symptomatic Conus lipomas: operative removal of the tumor (to decompress the dural sac and the exiting nerve roots) and untethering $[1,3,4]$. Such an intervention should prevent progression of the neurological symptoms or even improve already existing symptoms $[5,6]$. Pareses and bladder function disorders in particular show a positive course after resolution of the Conus base. There is lack of consensus to date about the treatment of asymptomatic lipomas of the Conus medullaris. The value of "prophylactic" operations has been viewed by some authors as completely outweighed by the operating risk taken on [8]. But since the introduction of intraoperative neurophysiological mapping and monitoring techniques into routine surgical procedures, there seems to be a moderately more positive opinion about early operative therapy, because such neurophysiological monitoring will hopefully be able to reduce the postoperative morbidity.

MEP is a way to intraoperatively test the function of the motor pathways. MEPs are described as generally reliable and safe to use for spinal indications [10,12-14]. This is confirmed by the technical success rate of $86.7 \%$ in the present study. A disadvantage of this monitoring technique though is that an influence on the potential can arise not only on the path between the operating area and the discharge musculature but also on the entire path between cranial potential evocation and the periphery. So beside various physiological influences, anesthesiological factors affecting the central nervous system have a major influence on the registered potentials. Finally, reversible and irreversible changes of the recorded potentials must be differentiated. Reversible changes in all cases accompany unchanged clinical symptomology or a rapid recovery of short-term functional compromise. These observations are comparable with those for cerebral operations in the central region $[18,19]$. Irreversible changes correlated with postoperative neurological deterioration in $7.7 \%$. So these potential changes have a prognostic value, especially since these predicted deteriorations did not improve within the first 3 months after surgery. Immediate clinical deteriorations in cases of reversibly changed MEPs showed a stable status or even improvements in the long-term clinical course. Thus these short-term clinical deteriorations can be best viewed as postoperative swelling phenomena. MEPs that did not change at any time during the operation predict with higher probability an unchanged or improving clinical outcome.

Although our study did show an acceptable successquota / responder-rate for the tibialis SEPs of $66.7 \%$, the sensitivity for mechanical maneuvers in the operating site was quite low, as already presented by Gunnarsson et al. [20]. Thus, anesthesiological influences have at least as much influence on the tibial SEPs as manipulations by the surgeon. This is partly explained also by the anatomical location of the sensory pathways. Manipulatory changes can therefore be expected at highest on the level of the rear part of the spinal cord - thus only for dorsal lipomas. For anatomical reasons, caudal and transitional Conus lipomas would be difficult to examine by means of tibial SEPs. Tibialis SEPs are also of limited value in the area of the exiting nerve roots. Here, only dermatome-specific SEPs would be available as an examination technique $[10,11,13,14]$, which requires a time investment that is unjustified in our view (both in regards to the number of stimulation sites and also due to the necessary time required for potential averaging). So consistent with most other studies on this topic $[10,11,13,14]$, dermatomespecific SEPs were not used in our study either.

It was technically feasible to carry out EMG in all cases, and there were no anesthesiological or physiological influences to be detected (having in mind, that no muscle relaxants were allowed in the course of surgery). Similar to the study of Valentini et al. [21], it was possible to better identify the neural structures of complex lesions, reducing the risk of surgical damage and incomplete detethering. In a study by Bindal \& Ghosh [17], electrophysiological feedback on the patient could be obtained in $76.2 \%$ of the cases through intraoperative EMG monitoring procedures for neurosurgical interventions. Our success rate of the initial nerve root identification of $60.2 \%$ was below the value reported by Bindal \& Ghosh [17], but the rate had reached $76.4 \%$ by the end of the operation, which is consistent with the literature.

The specific potential changes in the free-running EMG after mechanical maneuvers were especially helpful for the surgeon. So, main results of this work are that: a) spontaneous activity can be recorded from the examined muscle groups also without manipulation, and it has no pathological value, b) phasic "spikes"/"bursts" are evoked by direct contact of nerve tissue with operating instruments, and c) tonic "trains" can be elicited by stronger traction on the nerve fibers or partial damage. Since spikes/bursts were registered in all 30 cases, these appear to have no immediate pathological value. Yet if 
these turn into trains from stronger traction, then this can prognostically indicate a mechanical lesion as seen in other studies before [22]. Also, EMG trains appear to be more sensitive than MEPs, for the motor deteriorations that were reversible immediately or within 3 months postop, as they were all prefigured by train activity. There were even 4 cases where train activity left no immediate clinical harm, thus confirming that trains might have worked as a very sensitive warning signal in these cases - forcing the surgeon to immediately change the surgical strategy. This includes immediate reduction of compression, traction or stretching neural structures to reduce neuropraxia as well as stopping thermal/bipolar dissection and mechanical ligation to reduce ischemic damage. Furthermore, the temperature of the irrigation saline has to be checked routinely. A detailed analysis of train duration, frequency of train recurrence, or train amplitudes in a larger patient sample might provide better information.

Nerve tissue could be distinguished from non-nerval tissue (tumor tissue, connective tissue etc.) by triggered EMG/direct bipolar stimulation in $60.2 \%$ of cases right after opening of the dura. Preexisting pareses equal or less than BMRC grade 2/5 were one reason for lack of stimulation success. Tumor masses at the beginning of the operation were another reason, as they might have covered or suppressed the exiting nerve roots. The stimulation gains are due to the tumor removal and preparing free the tumor-covered nerve roots. However, anatomical liberation of tumor-covered nerve roots does not seem to correlate with improved post-op function. This may be due to the fact, that dislocated nerve roots still may work properly or non-working nerve roots will not regain function after free-preparation. Stimulation losses on the other hand appear to have the potential to forewarn of postoperative clinical deteriorations. Our findings are consistent with a previous study that found that the simultaneous use of EMG and MEP monitoring procedures is a reliable method for the differentiation of neural vs. fibrous or tumorous tissue [16].

There are many methodological difficulties in evaluating the optimal intraoperative monitoring procedures for surgery of spinal lipomas in the Conus medullaris. Looking exclusively at statistical parameters (such as sensitivity, specificity, or predictive value), the previously described discrepancies (e.g. about the value of SEPs) can be found in the literature. This is partly due to the fact that many studies do not differentiate between surgeries on the spinal cord, Conus, or Cauda, but instead evaluate them all together as "spinal operations". Studies that occupy themselves electrophysiologically only with Conus tumors are, like the present study, mostly rather small, so that valid statistical analysis is only possible with qualifications. Furthermore, the evaluation and analysis of the neurophysiological signals generated depends heavily on the experience of the investigators with the various techniques yet also on their preferences for one or another of them. Also, the prognostic and predictive explanatory power of observed signal changes can only be determined with qualifications, because changes in potential (which in part are arbitrarily defined differently by different research groups) are taken as warning signals and therefore have an immediate effect on the operative process. So the actual specifity of "train" activity in the free-running EMG (for example) remains unclear, because their intraoperative occurrence may lead directly to a consequence in the surgical strategy. Yet there is no way around this, because a scientifically motivated "wait-and-see" approach would be ethically indefensible. But now, with all these methodological caveats aside, what would be the most reliable (yet time and cost efficient) examination set-up, for intraoperative monitoring in the Conus medullaris?

In our viewpoint, focusing on the actual results of this retrospective study and according to the current literature, the following statements can be made:

a) The technical feasibility of free-running and triggered EMG is nearly 100\%; whereas, transcranial MEP and tibialis SEP are much more technically demanding.

b) The localization of nerve roots in the operating site can be carried out most reliably with the two EMG techniques.

c) The free-running EMG and the transcranial MEP are best adapted to being an intraoperative warning signal.

d) Tibialis SEPs appear to be only of secondary usefulness for interventions on the Conus medullaris, and it is questionable whether the use of dermatome-specific SEPs justifies the time involved.

e) In this retrospective study, motor signals had the best prognostic value, in descending order: triggered EMG, transcranial MEP, free-running EMG.

\section{Conclusions}

According to the results of this study, the authors favor a routine set-up of intraoperative neurophysiological monitoring for spinal lipomas in the area of the Conus medullaris consisting of transcranial evoked MEP, free-running EMG, and triggered EMG. This enables a site cartography at the beginning of the operation, continuous intraoperative functional testing (nerve vs. non-nerve tissue), and a prognostic evaluation about the expectable post-operative symptomology.

\section{Consent}

Written informed consent was obtained from all patients for the publication of this report and any accompanying images. For the seventeen patients below the legal age of 18 years, consent from the the patient's guardian/parent was obtained. 


\section{Competing interests}

The authors declare that they have no competing interests.

\section{Authors' contributions}

OS is main author and is responsible for study conception and design; SM, MAC, MC and SH have made substantial contributions to conception and design, data acquisition and analysis of data; OS and TK have been involved in drafting the manuscript and revising it critically for important intellectual content and have given final approval of the version to be published. All authors read and approved the final manuscript.

\section{Acknowledgments}

The authors would like to thank Michael Hanna, PhD, (Medical Manuscript Service, New York) for translating and editing the manuscript.

\section{Author details}

'Zentrum für Wirbelsäulenchirurgie und Neurotraumatologie, DRK Kliniken Berlin Westend, Spandauer Damm 130, 14050 Berlin, Germany.

${ }^{2}$ Neurochirurgische Klinik, Charité-Universitätsmedizin Berlin, Berlin, Germany. ${ }^{3}$ Interdisziplinäres Wirbelsäulenzentrum, Vivantes Auguste-Viktoria-Klinikum, Berlin, Germany. ${ }^{4}$ Neurochirurgie, Vivantes Klinikum im Friedrichshain, Berlin, Germany. ${ }^{5}$ Abteilung für Neurochirurgie, Schlosspark-Klinik, Berlin, Germany.

Received: 9 June 2014 Accepted: 19 August 2014

Published: 2 September 2014

\section{References}

1. Blount JP, Elton S: Spinal lipomas. Neurosurg Focus 2001, 10:e3.

2. Harrison MJ, Mitnick RJ, Rosenblum BR, Rothman AS: Leptomyelolipoma: analysis of 20 cases. J Neurosurg 1990, 73:360-367.

3. Xenos C, Sgouros S, Walsh R, Hockley A: Spinal lipomas in children. Pediatr Neurosurg 2000, 32:295-307.

4. Arai H, Sato K, Okuda O, Miyajima M, Hishii M, Nakanishi H, Ishii H: Surgical experience of 120 patients with lumbosacral lipomas. Acta Neurochir (Wien) 2001, 143:857-864.

5. Dorward NL, Scatliff JH, Hayward RD: Congenital lumbosacral lipomas: pitfalls in analysing the results of prophylactic surgery. Childs Nerv Syst 2002, 18:326-332.

6. Morimoto K, Takemoto O, Wakayama A: Spinal lipomas in children-surgical management and long-term follow-up. Pediatr Neurosurg 2005, 41:84-87.

7. Constantini S, Miller DC, Allen JC, Rorke LB, Freed D, Epstein FJ: Radical excision of intramedullary spinal cord tumors: surgical morbidity and long-term follow-up evaluation in 164 children and young adults. J Neurosurg 2000, 93(2 Suppl):183-193.

8. Kulkarni AV, Pierre-Kahn A, Zerah M: Conservative management of asymptomatic spinal lipomas of the conus. Neurosurgery 2004, 54:868-873.

9. Kothbauer KF: Intraoperative neurophysiologic monitoring for intramedullary spinal-cord tumor surgery. Neurophysiol Clin 2007, 37:407-414.

10. Nagle KJ, Emerson RG, Adams DC, Heyer EJ, Roye DP, Schwab FJ, Weidenbaum M, McCormick P, Pile-Spellman J, Stein BM, Farcy JP, Gallo EJ, Dowling KC, Turner CA: Intraoperative monitoring of motor evoked potentials: a review of 116 cases. Neurology 1996, 47:999-1004.

11. Calancie B, Harris W, Brindle GF, Green BA, Landy HJ: Threshold-level repetitive transcranial electrical stimulation for intraoperative monitoring of central motor conduction. J Neurosurg 2001, 95(2 Suppl):161-168.

12. de Haan P, Kalkman CJ: Spinal cord monitoring: somatosensory- and motor-evoked potentials. Anesthesiol Clin North America 2001, 19:923-945.

13. Pelosi L, Lamb J, Grevitt M, Mehdian SM, Webb JK, Blumhardt LD: Combined monitoring of motor and somatosensory evoked potentials in orthopaedic spinal surgery. Clin Neurophysiol 2002, 113:1082-1091.

14. Costa P, Bruno A, Bonzanino M, Massaro F, Caruso L, Vincenzo I, Ciaramitaro $P$, Montalenti E: Somatosensory- and motor-evoked potential monitoring during spine and spinal cord surgery. Spinal Cord 2007, 45:86-91.

15. Bose B, Wierzbowski LR, Sestokas AK: Neurophysiologic monitoring of spinal nerve root function during instrumented posterior lumbar spine surgery. Spine 2002, 27:1444-1450.

16. von Koch CS, Quinones-Hinojosa A, Gulati M, Lyon R, Peacock WJ, Yingling CD: Clinical outcome in children undergoing tethered cord release utilizing intraoperative neurophysiological monitoring. Pediatr Neurosurg 2002, 37:81-86.
17. Bindal RK, Ghosh S: Intraoperative electromyography monitoring in minimally invasive transforaminal lumbar interbody fusion. J Neurosurg Spine 2007, 6:126-132.

18. Kombos T, Suess O, Ciklatekerlio Ö, Brock M: Monitoring of intraoperative motor evoked potentials to increase the safety of surgery in and around the motor cortex. J Neurosurg 2001, 95:608-614.

19. Suess O, Suess S, Brock M, Kombos T: Intraoperative electrocortical stimulation of brodman area 4: A 10-year analysis of 255 cases. Head Face Med 2006, 2:20. 03July2006.

20. Gunnarsson T, Krassioukov AV, Sarjeant R, Fehlings MG: Real-time continuous intraoperative electromyographic and somatosensory evoked potential recordings in spinal surgery: correlation of clinical and electrophysiologic findings in a prospective, consecutive series of 213 cases. Spine 2004, 29:677-684.

21. Valentini LG, Visintini S, Mendola C, Casali C, Bono R, Scaioli W, Solero CL: The role of intraoperative electromyographic monitoring in lumbosacral lipomas. Neurosurgery 2005, 56:315-323.

22. Suess $\mathrm{O}$, Brock M, Kombos T: Motor nerve root monitoring during percutaneous transforaminal sequestrectomy under general anestheia for intra- and extraforaminal lumbar disc herniation. Zentralb/ Neurochir 2005, 66:190-201.

\section{doi:10.1186/s13037-014-0035-4}

Cite this article as: Suess et al:: The value of intraoperative

neurophysiological monitoring for microsurgical removal of conus medullaris lipomas: a 12-year retrospective cohort study. Patient Safety in Surgery $20148: 35$

\section{Submit your next manuscript to BioMed Central and take full advantage of:}

- Convenient online submission

- Thorough peer review

- No space constraints or color figure charges

- Immediate publication on acceptance

- Inclusion in PubMed, CAS, Scopus and Google Scholar

- Research which is freely available for redistribution 